\title{
Impact of Antenna Selection on Cognitive Radio System Capacity
}

\author{
Pawel A. Dmochowski*, Peter J. Smith ${ }^{\dagger}$, Mansoor Shafi ${ }^{\ddagger}$, and Himal A. Suraweera ${ }^{\S}$ \\ * School of Engineering and Computer Science, Victoria University of Wellington, Wellington, New Zealand \\ $\dagger$ Department of Electrical and Computer Engineering, University of Canterbury, Christchurch, New Zealand \\ $\ddagger$ Telecom New Zealand, Wellington, New Zealand \\ $\S$ Engineering Product Development, Singapore University of Technology and Design, Singapore \\ Email:pdmochowski@ieee.org,p.smith@elec.canterbury.ac.nz,mansoor.shafi@telecom.co.nz, himalsuraweera@sutd.edu.sg
}

\begin{abstract}
We examine the effect of transmit antenna selection on the secondary user ( $\mathrm{SU}$ ) and primary user (PU) capacity in a multiple-input single-output cognitive radio system. We consider antenna selection with the aim of improving SU and PU capacity and mitigating the effects of incomplete channel knowledge by the SU. Under a minimum PU signal-to-interference-and-noise ratio constraint, analytical and semi-analytical expressions for the permissible SU transmit power are derived, which are used in obtaining capacity cumulative distribution functions by means of simulations. We show that PU antenna selection is effective at reducing the $\mathrm{SU}$ blocking probability, the probability that it cannot transmit, while SU antenna selection leads to significant gains in its capacity. Antenna selection at the $\mathrm{SU}$ is also shown to effectively compensate for incomplete channel knowledge and can be used to achieve moderate improvements in PU capacity.
\end{abstract}

\section{INTRODUCTION}

Cognitive radio (CR) [1] is a communication paradigm in which secondary (cognitive) users (SUs) can access parts of the primary user (PU) spectrum for their transmission, provided they cause minimal or no interference to the PUs [2]. A number of papers have appeared on various aspects of CR systems, including information theoretic capacity limits (see, for eg., [3]-[7]). The SUs can protect the PU transmissions by regulating their transmitter power so that average/peak interference to the PU receiver remains below a threshold [4].

Regulating the interference to the PU-Rx requires a knowledge of the channel state information (CSI) of the SU-Tx to PU-Rx link. In [6], we evaluated the impact of outdated/quantized CSI on SU capacity. It was assumed that the SU-Tx only has partial information about the SU-Tx to PU-Rx link. As such, a conservative approach in determining the interference caused at the PU-Rx is required. Due to the outdated CSI the interference threshold cannot be guaranteed all the time; thus, based on the interference cumulative distribution function (cdf), a probabilistic threshold margin was used.

The signal-to-noise ratio (SNR) at the PU-Rx is variable due to fading on the PU link. In order to include these signal variations, it is best to model interference to the PU receiver via a minimum signal-to-interference noise ratio (SINR) constraint, beyond which degradation is not accepted. When the PU SNR is below this level then the SU cannot transmit. The SINR constraint allows the interference level to vary according to the level of the PU-Tx to PU-Rx link. The price of this relaxation is that information about the CSI of the PU-Tx to PU-Rx link must be available to the SU-Tx.
In [7] we consider the SINR constraint and examine its impact on SU capacity under different levels of CSI knowledge. We derived SU capacity cdfs for single antenna links and have shown that the absence of CSI severely limits the SU transmission opportunities under realistic operating conditions.

Existing antenna selection literature, e.g., [8]-[11] considers selection at the SU-Tx and assumes perfect CSI. In this work, we explore antenna selection at both the SU-Tx and PU-Tx and consider the impact of having precise or imperfect channel knowledge of the various constituent links. We also consider the impact of PU interference at the SU-Rx, where we explore three possible benefits of antenna selection: improving the SU and PU capacities, and counteracting the SU performance degradation resulting from incomplete knowledge of various channel links. We show that SU capacity is strongly affected by periods of time when the SU cannot transmit due to low SNR on the PU-Rx. The probability of this event (known as $S U$-blocking) can be significantly reduced with antenna selection on the PU link. We show that for the cases considered, one additional antenna at the SU-Tx can be used to compensate for incomplete channel knowledge. We derive analytical and semi-analytical expressions for the allowed SU transmit power, and the probabilities or conditions for SU blocking. The model complexity prevents the derivation of closed form SU capacity cdfs. Instead, these are obtained by means of Monte-Carlo simulations using the transmit power values derived.

\section{SySTEM MODEL}

Consider a CR system in Fig. 1, with the SU-Tx and PU-Tx transmitting simultaneously over a common frequency channel to their respective receivers. We assume MISO SU and PU links, with the users selecting one of $N$ and $M$ antennas for transmission, respectively. Independent point-to-point flat Rayleigh fading channels are assumed. Let $g_{p}^{(j)}=\left|h_{p}^{(j)}\right|^{2}$, $g_{p s}^{(j)}=\left|h_{p s}^{(j)}\right|^{2}, g_{s}^{(i)}=\left|h_{s}^{(i)}\right|^{2}$, and $g_{s p}^{(i)}=\left|h_{s p}^{(i)}\right|^{2}$ denote the instantaneous channel gains of the PU-Tx to PU-Rx, PUTx to SU-Rx, SU-Tx to SU-Rx and SU-Tx to PU-Rx links, respectively, with the channel indexes $i=1, \ldots, N$ and $j=$ $1, \ldots, M$. We will denote the exponential probability density functions (pdfs) of the random variables (RVs) $g_{p}^{(j)}, g_{s}^{(i)}, g_{p s}^{(j)}$, and $g_{s p}^{(i)}$ by $f_{g_{p}}(x), f_{g_{s}}(x), f_{g_{p s}}(x)$ and $f_{g_{s p}}(x)$, respectively. These distributions are governed by their corresponding parameters $\Omega_{p}=\mathbb{E}\left(g_{p}^{(j)}\right), \Omega_{s}=\mathbb{E}\left(g_{s}^{(i)}\right), \Omega_{s p}=\mathbb{E}\left(g_{s p}^{(i)}\right)$ and $\Omega_{p s}=\mathbb{E}\left(g_{p s}^{(j)}\right)$, where $\mathbb{E}(\cdot)$ denotes the expectation operator. 


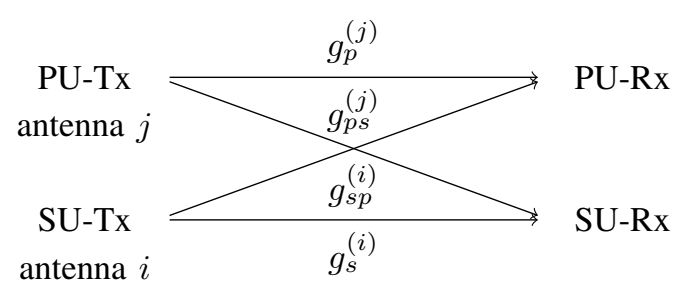

Fig. 1. System model.

We consider the impact of antenna selection on the SU and PU capacities, denoted respectively by $C_{S U}$ and $C_{P U}$, with the following aims

- Scenario A: improving the SU capacity.

- Scenario B: combating the lack of CSI, specifically when the SU-Tx knows only the means $\Omega_{p}$ and/or $\Omega_{s p}$.

- Scenario $C$ : improving the PU capacity by reducing the SU interference at the PU-Tx.

In our model, we impose a constraint on the minimum SINR, $\gamma_{p}$, at the PU-Rx, that is

$$
\gamma_{p}=\frac{P_{p} g_{p}^{(m)}}{P_{s} g_{s p}^{(n)}+\sigma_{p}^{2}}, \text { and } \gamma_{p} \geq \gamma_{T},
$$

where $\gamma_{T}$ is a predefined SINR threshold, $P_{p}$ is the PU transmit power, $P_{s}$ is the permissible $\mathrm{SU}$ transmit power, and $\sigma_{p}^{2}$ is the additive white Gaussian noise (AWGN) variance at the PU-Rx. Superscripts $m$ and $n$ denote the indices of the selected PU-Tx and SU-Tx antennas, respectively. The former is chosen by the PU to maximize the PU link gain, that is

$$
m=\underset{j=1, \ldots, M}{\arg \max } g_{p}^{(j)} .
$$

Note that if the PU-Rx SNR, $P_{p} g_{p}^{(m)} / \sigma_{p}^{2}<\gamma_{T}$, the SINR constraint in (1) cannot be satisfied, and thus the SU transmit power is zero. We will refer to this condition as SU blocking. In addition, we impose a maximum SU transmit power constraint, denoted by $P_{m}$, which arises in practice, for example, due to power amplifier nonlinearities. A similar constraint exists for the PU transmit power. Thus, in scenarios where the SU-Tx knows $g_{p}^{(m)}$ the actual SU transmit power is given by

$$
P_{t}= \begin{cases}0 & \frac{P_{p} g_{p}^{(m)}}{\gamma_{T}}<\sigma_{p}^{2} \\ \min \left(P_{s}, P_{m}\right) & \text { otherwise }\end{cases}
$$

where $P_{s}$ is obtained from (1) by solving $\gamma_{T}=\gamma_{p}$. As will be shown in Section III, the $P_{t}=0$ condition for some of the cases in Scenario B will depend on the choice of system parameters and thus (3) will be modified accordingly.

We note that the above constraints can only be guaranteed if the SU-Tx has knowledge of $g_{p}^{(j)}$ and $g_{s p}^{(i)}$, that is under Scenarios $A$ and $C$. As a result, in analysing Scenario $B$ we use probabilistic constraints, where we require the SINR constraint to hold with an acceptably high probability, $1-\alpha$, where $\alpha$ is small. These are described in detail in Section III-B.

The SU and PU capacities are given by

$$
C_{S U}=\log _{2}\left(1+\frac{P_{t} g_{s}^{(n)}}{\sigma_{s}^{2}+P_{p} g_{p s}^{(m)}}\right),
$$

$$
C_{P U}=\log _{2}\left(1+\frac{P_{p} g_{p}^{(m)}}{\sigma_{p}^{2}+P_{t} g_{s p}^{(n)}}\right),
$$

where $\sigma_{s}^{2}$ is the AWGN variance at the SU-Rx.

The main system variables are parameterized as in [7], that is using

$$
c_{1}=\frac{\Omega_{s p}}{\Omega_{s}},
$$

which represents the ratio of SU interference to desired $\mathrm{SU}$ channel strength, and

$$
c_{2}=\frac{\gamma_{T}}{P_{p} \Omega_{p} / \sigma_{p}^{2}},
$$

which is the ratio of the minimum target SINR to the actual SNR at the PU-Rx. Thus, increasing $c_{2}$ corresponds to reducing the allowable interference.

In what follows, we describe in detail the scenarios considered, deriving in each case the permissible SU transmit power, $P_{s}$, under the SINR constraint (1).

\section{SU AND PU CAPACITY}

\section{A. Scenario A}

The SU-Tx performs antenna selection to increase its capacity. It selects the antenna which maximizes the received signal strength at the SU-Rx based on the gain $g_{s}^{(i)}$ and the transmit powers satisfying the SINR constraint. Hence,

$$
n=\underset{i=1, \ldots, N}{\arg \max } P_{t}^{(i)} g_{s}^{(i)},
$$

where each possible transmit power, $P_{t}^{(i)}$, is given by (3) as

$$
P_{t}^{(i)}=\left\{\begin{array}{ll}
0 & \frac{P_{p} g_{p}^{(m)}}{\gamma_{T}}<\sigma_{p}^{2} \\
\min \left(P_{s}^{(i)}, P_{m}\right) & \text { otherwise }
\end{array},\right.
$$

and $P_{s}^{(i)}$ is obtained by letting $\gamma_{p}=\gamma_{T}$ in (1), giving

$$
P_{s}^{(i)}=\left(\frac{P_{p} g_{p}^{(m)}}{\gamma_{T}}-\sigma_{p}^{2}\right) \frac{1}{g_{s p}^{(i)}} .
$$

In (9) and (10) it is assumed that the PU selects its antenna based on (2). The SU capacity is computed from (4), with the SU transmit power given by (9), where for notational simplicity we let $P_{t} \triangleq P_{t}^{(n)}$ with $n$ given by (8). To derive the SU blocking probability we use (3) and (7) to give

$$
\operatorname{Pr}\left\{P_{t}=0\right\}=\operatorname{Pr}\left\{g_{p}^{(m)}<c_{2} \Omega_{p}\right\} .
$$

Since $g_{p}^{(m)}$ is the maximum order statistic of $M$ independent exponential random variables, the cdf in (11) is given by

$$
\operatorname{Pr}\left\{P_{t}=0\right\}=\left(1-e^{-c_{2}}\right)^{M} .
$$

\section{B. Scenario $B$}

The SU-Tx utilizes antenna selection with the aim of reducing the effects of incomplete knowledge of the channel gains $g_{p}^{(j)}$ and $g_{s p}^{(i)}$. Specifically, we consider the case where only the means $\Omega_{p}$ and/or $\Omega_{s p}$ are available, and thus the SINR constraint can only be satisfied with some probability $1-\alpha$. We present three sub-cases. 
1) Scenario B1 $-g_{p}^{(j)}, \Omega_{s p}$ known: ${ }^{1}$ In this case the CR target is to satisfy (1) with a probability $1-\alpha$. Hence,

$$
\operatorname{Pr}\left\{\frac{P_{p} g_{p}^{(m)}}{P_{s} g_{s p}^{(n)}+\sigma_{p}^{2}} \geq \gamma_{T} \mid g_{p}^{(m)}, \Omega_{s p}\right\}=1-\alpha,
$$

where the optimum PU channel is selected using (2). We use the cdf of $g_{s p}^{(n)}$ to compute (13) and solving for $P_{s}$ obtain

$$
P_{s}=-\frac{P_{p} g_{p}^{(m)}-\gamma_{T} \sigma_{p}^{2}}{\ln (\alpha) \gamma_{T} \Omega_{s p}} .
$$

Here, due to the lack of knowledge of $g_{s p}^{(i)}$ the SU-Tx is unable to compute the permissible powers, $P_{t}^{(i)}$, and thus antenna selection is performed based on the link gains $g_{s}^{(i)}$, that is

$$
n=\underset{i=1, \ldots, N}{\arg \max } g_{s}^{(i)} .
$$

The SU capacity is then computed from (4), with the SU transmit power given by (3) and (14), with $n$ given by (15).

We note that the zero transmission probability for Scenario $B 1$ is given by (12) derived for Scenario $A$.

2) Scenario $B 2-g_{s p}^{(i)}, \Omega_{p}$ known: Here, the probabilistic SINR constraint is given by

$$
\operatorname{Pr}\left\{\frac{P_{p} g_{p}^{(m)}}{P_{s}^{(i)} g_{s p}^{(i)}+\sigma_{p}^{2}} \geq \gamma_{T} \mid g_{s p}^{(i)}, \Omega_{p}\right\}=1-\alpha,
$$

which gives

$$
\operatorname{Pr}\left\{g_{p}^{(m)} \leq \frac{\gamma_{T}\left(P_{s}^{(i)} g_{s p}^{(i)}+\sigma_{p}^{2}\right)}{P_{p}} \mid g_{s p}^{(i)}, \Omega_{p}\right\}=\alpha .
$$

As in (11), using the cdf of the maximum order statistic of $M$ independent exponential variables, solving (17) gives

$$
P_{s}^{(i)}=-\left(\frac{\ln \left(1-\alpha^{1 / M}\right) P_{p} \Omega_{p}}{\gamma_{T}}+\sigma_{p}^{2}\right) \frac{1}{g_{s p}^{(i)}},
$$

giving

$$
P_{t}^{(i)}=\max \left\{0, \min \left\{P_{m}, P_{s}^{(i)}\right\}\right\} .
$$

The SU-Tx can maximize the received signal strength according to (8) and the SU capacity is found using (19) and (4).

The zero SU transmission condition is found by solving for the system parameters resulting in a negative $P_{s}^{(i)}$ in (18). Rearranging (18) shows that the SU will fail to satisfy the SINR constraint when

$$
c_{2}>-\ln \left(1-\alpha^{1 / M}\right) \text {. }
$$

3) Scenario $B 3-\Omega_{s p}$ and $\Omega_{p}$ known: Following a similar approach to Scenarios $B 1$ and $B 2$ gives

$$
\operatorname{Pr}\left\{\frac{P_{p} g_{p}^{(m)}}{P_{s} g_{s p}^{(n)}+\sigma_{p}^{2}} \geq \gamma_{T} \mid \Omega_{p}, \Omega_{s p}\right\}=1-\alpha .
$$

Conditioning on $g_{s p}^{(n)}$ one can show that

$$
\mathbb{E}\left\{\left(1-e^{-\frac{\gamma_{T}\left(P_{s} g_{s p}^{(n)}+\sigma_{p}^{2}\right)}{P_{p} \Omega_{p}}}\right)^{M}\right\}=\alpha .
$$

\footnotetext{
${ }^{1} \mathrm{As}$ it is assumed that these links are estimated by the PU-Rx, it is reasonable to consider a case of more precise knowledge of $g_{p}^{(j)}$ than $g_{s p}^{(i)}$.
}

Binomial expansion of the power term in (22) gives

$$
\alpha=\sum_{k=0}^{M}\left(\begin{array}{c}
M \\
k
\end{array}\right) \frac{(-1)^{k} e^{-\frac{k \gamma_{T} \sigma_{p}^{2}}{P_{p} \Omega_{p}}} P_{p} \Omega_{p}}{P_{p} \Omega_{p}+k \Omega_{s p} P_{s}} .
$$

To the authors' knowledge, no analytical solution for $P_{s}$ in (23) exists and a numerical approach is required. We note that $P_{t}$ is dependent only on the system parameters,

$$
P_{t}= \begin{cases}0 & P_{s}<0 \\ P_{s} & 0<P_{s}<P_{m} \\ P_{m} & P_{s}>P_{m}\end{cases}
$$

Blocking occurs when $P_{s}$, obtained from (23), is negative.

Finally, $C_{S U}$ is obtained by substituting (24) into (4) with indices $m$ and $n$ determined via (2) and (15), respectively.

\section{Scenario $C$}

We consider SU-Tx antenna selection with the aim of reducing the interference to the PU-Rx. The SU reduces its transmit power such that it achieves an average capacity equivalent to that of a SISO link. We assume the SU-Tx has a knowledge of $g_{p}^{(j)}$ and $g_{s p}^{(i)}$, and thus it is able to compute the achievable capacities for each of its transmit antennas,

$$
C_{S U}^{(i)}=\log _{2}\left(1+\frac{P_{t}^{(i)} g_{s}^{(i)}}{\sigma_{s}^{2}+P_{p} g_{p s}^{(m)}}\right),
$$

where $P_{t}^{(i)}$ is computed from (9) and (10), and the PU selected antenna is given by (2). The SU selects the antenna corresponding to the highest instantaneous capacity,

$$
n=\underset{i=1, \ldots, N}{\arg \max } C_{S U}^{(i)} .
$$

Up to this point, the SU is seeking to optimize its capacity. However, once the best antenna is selected, the SU then backs off its transmit power to reduce the interference to the PU. In doing this the SU does not want to reduce power too far. In particular its target is to not drop below the mean capacity of a SISO link. Hence, the SU-Tx reduces the transmit power to $P_{t}^{\prime}$ such that the $\mathrm{SU}$ capacity using antenna $n$ equals the average capacity offered by the $N$ antennas,

$$
\bar{C}_{S U}=\frac{1}{N} \sum_{i} C_{S U}^{(i)}=\log _{2}\left(1+\frac{P_{t}^{\prime} g_{s}^{(n)}}{\sigma_{s}^{2}+P_{p} g_{p s}^{(m)}}\right) .
$$

Solving (27) gives

$$
P_{t}^{\prime}=\left(2^{\bar{C}_{S U}}-1\right) \frac{\sigma_{s}^{2}+P_{p} g_{p s}^{(m)}}{g_{s}^{(n)}} .
$$

The SU and PU capacities are given by (4) and (5) with $P_{t}^{\prime}$ used in place of $P_{t}$.

Finally, we note that the zero transmission probability for Scenario $C$ is governed by (12). 


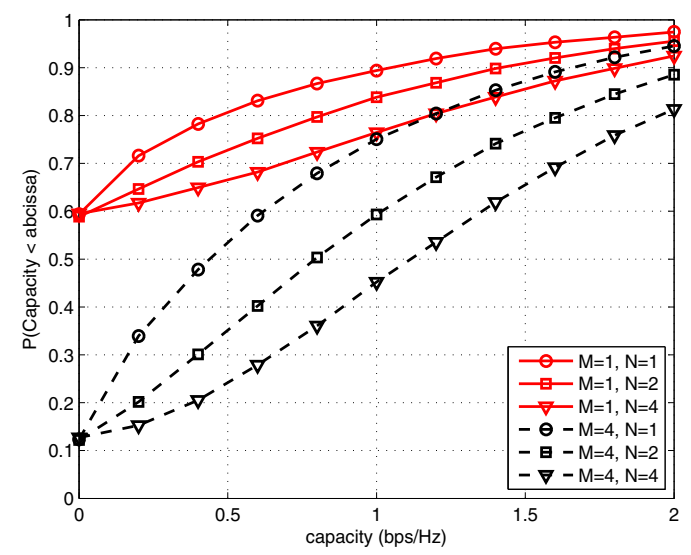

Fig. 2. Scenario A: $C_{S U}$ cdfs $\left(c_{1}=0.3, c_{2}=0.9\right)$

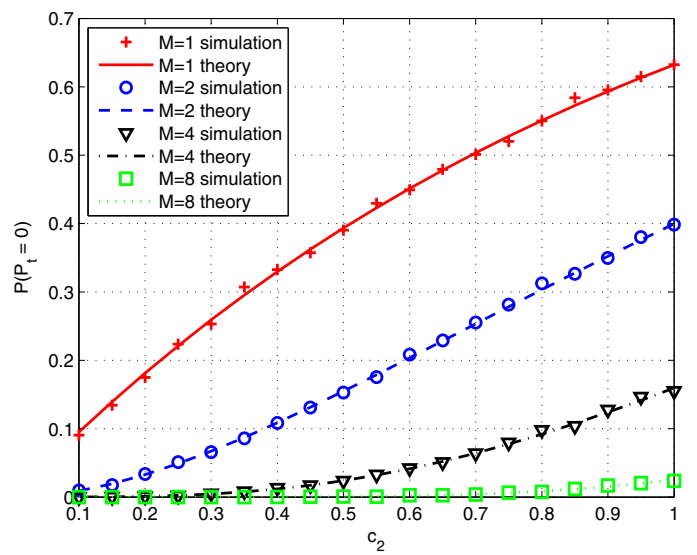

Fig. 3. SU blocking probability - Scenarios $A, B 1$ and $C$.

\section{Simulation Results}

We present simulation results describing the capacity performance under the scenarios described in Section III. In all cases we have normalized the powers so that $P_{p} / \sigma_{p}^{2}=P_{m} / \sigma_{s}^{2}=0$ $\mathrm{dB}$ and $\Omega_{p} / \sigma_{p}^{2}=\Omega_{s} / \sigma_{s}^{2}=5 \mathrm{~dB}$, where we have assumed $\sigma_{p}^{2}=\sigma_{s}^{2}$. In Scenario $B$ we set $\alpha=0.1$. In all simulations, unless otherwise stated, $c_{1}=0.3$ and $c_{2}=0.9$, which corresponds to relatively strict requirements placed on the SU.

\section{A. Scenario A}

Figure 2 shows the SU capacity cdfs under Scenario A simulated using (4) with SU-Tx power governed by (9) and (10). The capacity improvement is mainly influenced by the number of PU-Tx antennas, as this dictates the probability of $\mathrm{SU}$ transmission. For $M=4$, increasing SU-Tx antennas to 2 and 4 increases the median capacity by approximately 0.4 and $0.8 \mathrm{bps} / \mathrm{Hz}$, respectively, as expected.

As demonstrated by Fig. 2, the dominating factor for the capacity cdf is the SU blocking probability due to the low PU SNR relative to $\gamma_{T}$. Figure 3 shows this probability as a function of $c_{2}$. This applies to Scenarios $A, B 1$ and $C$. Simulated and theoretical values from (12) are plotted for varying $M$. We note that the curves are independent of the number of SU-Tx antennas, $N$, and the parameter $c_{1}$.

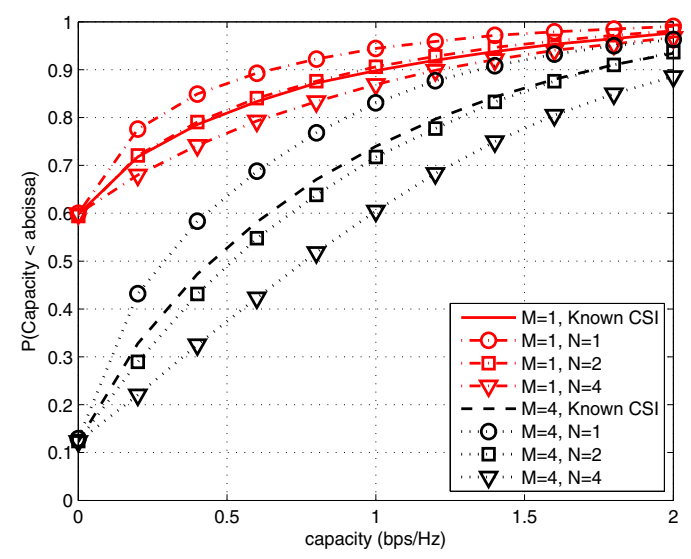

Fig. 4. Scenario B1: $C_{S U}$ cdfs $\left(c_{1}=0.3, c_{2}=0.9\right)$.
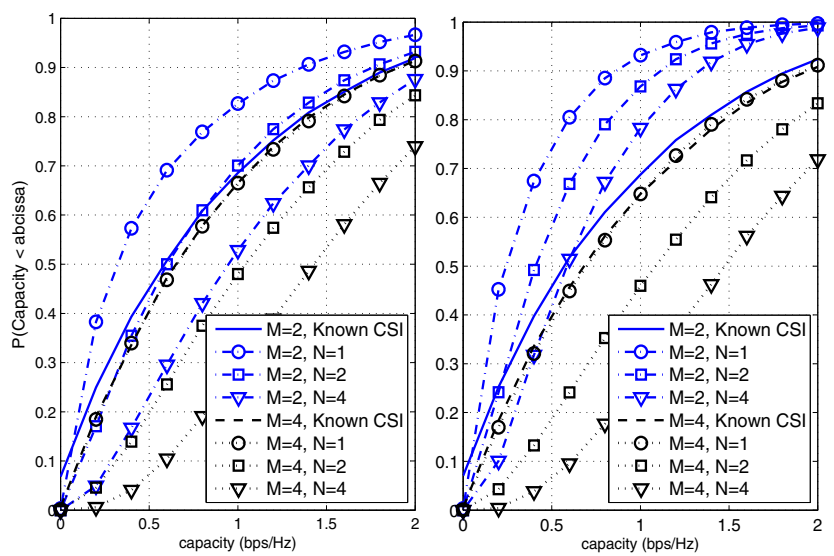

Fig. 5. Scenarios $B 2$ and B3: $C_{S U} \operatorname{cdfs}\left(c_{1}=0.3, c_{2}=0.3\right)$.

\section{B. Scenario B}

Figure 4 shows the $C_{S U}$ cdfs under Scenario B1 as obtained from (4) with transmit power determined by (14) and (9). As discussed in Section II, the aim is to use antenna selection to compensate for the lack of CSI, in this case that of $g_{s p}^{(i)}$. The target cdfs (plotted with bold lines in Fig. 4) are thus obtained from Scenario A. For $M=1$ and $M=4$ two SU-Tx antennas are required to compensate for incomplete knowledge of $g_{s p}^{(i)}$, with additional antennas providing further gain. The improvement from $M=1$ to $M=4$ is expected as increasing $M$ simply increases the PU channel strength.

In Scenarios B2 and B3, the parameters $c_{1}=0.3$ and $c_{2}=$ 0.9 are too restrictive to allow the SU-Tx to transmit. This is consistent with the findings in [7], where it was shown that the knowledge of the PU-Tx to PU-Rx link is more valuable to the SU than the SU-Tx to PU-Rx link. The results are thus obtained for the relaxed value of $c_{2}=0.3$. Figure 5 shows the $C_{S U}$ cdfs for $M=2,4$, when $\mathrm{SU}$ transmission is possible. For Scenario B2, we observe that for $M=2$ two SU-Tx antennas are required to compensate for imperfect knowledge of $g_{p}^{(j) 2}$. For $M=4$, when the PU has a strong link, the SU-Tx is able to transmit at the maximum level $P_{m}$. Thus, the performance

\footnotetext{
${ }^{2}$ For a stricter probabilistic SINR constraint, i.e. $\alpha<0.1$, more antennas are required, assuming no SU blocking occurs.
} 


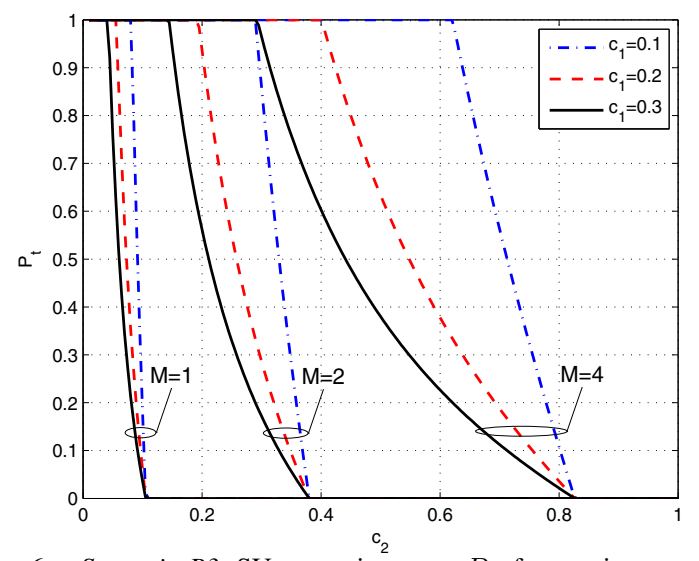

Fig. 6. Scenario B3: SU transmit power, $P_{t}$, for varying $c_{1}$ and $c_{2}$.

is the same as for Scenario $A$ for $M=4^{3}$. In the case of Scenario B3, the curves were obtained by numerically solving for $P_{s}$ in (23), which was subsequently used to determine the actual transmit power from (24) and the capacity via (4). For $M=2$ PU-Tx antennas, the limitations of Scenario B3 necessitate $N=4$ or more SU antennas to compensate for the lack of knowledge of both $g_{p}^{(j)}$ and $g_{s p}^{(i)}$. The results are significantly different for $M=4$, where the added diversity provided to the $\mathrm{PU}$ allows for maximum $\mathrm{SU}$ transmit power $P_{t}=P_{m}=1$. This is confirmed by the results in Fig. 6, noting the $c_{1}=0.3$ curves for $M=2$ and $M=4$ at $c_{2}=0.3$. The limiting case of $P_{t}=P_{m}$ results in the same performance of Scenarios $A$ and $B 3$, as evidenced by the plots in Fig 5. As noted in Section III-B3, the transmit power, $P_{t}$, in Scenario $B 3$ is determined entirely by the choice of system parameters, which determines the outcome of (24). Figure 6 shows the resulting $P_{t}$ values for a range of $c_{1}$ and $c_{2}$. The results show that when operating under demanding conditions (i.e., $c_{2}>$ $0.5)$ the SINR constraint cannot be satisfied with a probability of $1-\alpha=0.9$ unless the PU is equipped with $M=4$ branches thus ensuring a strong link to the PU-Rx.

\section{Scenario $C$}

Figure 7 shows $C_{P U}$ cdfs, including reference capacity cdfs achievable in the absence of SU transmission.

Referring to Fig. 7, we note that in the high capacity regime, the SU-Tx with $N=4$ antennas is able to reduce by half the capacity loss caused to the primary, specifically from a loss of $1.2 \mathrm{bps} / \mathrm{Hz}$ to $0.6 \mathrm{bps} / \mathrm{Hz}$. In the low capacity region, corresponding to weak $g_{p}^{(m)}$, the SU-Tx is not permitted to transmit, and thus all $C_{P U}$ cdfs overlap with the reference curves of no SU interference. There exists some threshold value of $g_{p}^{(m)}$ (independent of $N$ ) and a corresponding threshold capacity value at which the SU-Tx commences transmission, resulting in a sudden drop in $C_{P U}$ as observed in Fig. 7. This jump is most pronounced for $N=1$ where the SU-Tx is not able to use antenna selection to reduce its interference at the PU-Tx.

\footnotetext{
${ }^{3}$ While not shown due to space constraints, similar results are obtained for Scenario $B 1$ for $M=4$ and $c_{2}=0.3$
}

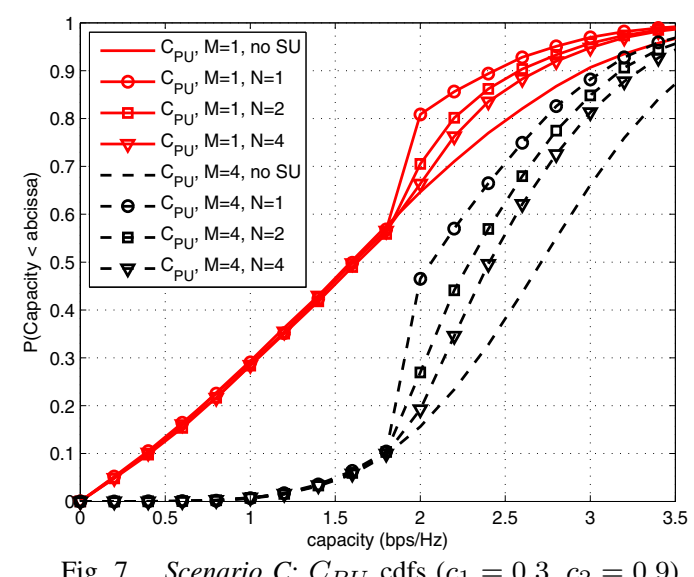

Fig. 7. Scenario $C$ : $C_{P U}$ cdfs $\left(c_{1}=0.3, c_{2}=0.9\right)$

\section{COnClusions}

We examined the impact of transmit antenna selection on the SU and PU capacity in a CR system. Three uses of antenna selection were considered, two aiming at improving SU and PU performance, and one to compensate for the lack of SUTx CSI. We derived expressions for the permissible transmit power under a SINR constraint, which were used to obtain capacity cdfs. SU capacity is dominated by the probability of zero transmission, and, as expected, antenna selection by the PU-Tx was shown to effectively reduce this probability. SUTx antenna selection provided additional gains in capacity. The SU antenna selection was shown to compensate for incomplete CSI, with one additional antenna sufficient in most cases considered. Finally, we showed that SU-Tx antenna selection can be used to reduce the PU capacity loss by a factor of two.

\section{REFERENCES}

[1] J. Mitola III, "Cognitive radio: An integrated agent architecture for software defined radio," Ph.D. dissertation, KTH, Sweden, May 2000.

[2] T. A. Weiss and F. K. Jondral, "Spectrum pooling: An innovative strategy for the enhancement of spectrum efficiency," IEEE Commun. Mag., vol. 42, pp. 8-14, March 2004.

[3] S. A. Jafar and S. Srinivasa, "Capacity limits of cognitive radio with distributed and dynamic spectral activity," IEEE J. Sel. Areas Commun., vol. 25, pp. 529-537, April 2007.

[4] A. Ghasemi and E. S. Sousa, "Fundamental limits of spectrum-sharing in fading environments," IEEE Trans. Wireless Commun., vol. 6, pp. 649-658, February 2007.

[5] R. Zhang and Y.-C. Liang, "Exploiting multi-antennas for opportunistic spectrum sharing in cognitive radio networks," IEEE J. Select. Topics in Signal Processing, vol. 2, pp. 88-102, Feb 2008.

[6] H. A. Suraweera, P. J. Smith, and M. Shafi, "Capacity limits and performance analysis of cognitive radio with imperfect channel knowledge," IEEE Trans. Veh. Technol., vol. 59, pp. 1811-1822, May 2010.

[7] P. A. Dmochowski, H. A. Suraweera, P. J. Smith, and M. Shafi, "Impact of channel knowledge on cognitive radio system capacity," in Proc. IEEE VTC2010-Fall, September 2010, pp. 1-5.

[8] M. F. Hanif and P. J. Smith, "On MIMO cognitive radios with antenna selection," in Proc. IEEE WCNC 2010, April 2010, pp. 1-6.

[9] H. Wang, J. Lee, S. Kim, and D. Hong, "Capacity enhancement of secondary links through spatial diversity in spectrum sharing," IEEE Trans. Wireless Commun., vol. 9, pp. 494-499, February 2010.

[10] J. Zhou and J. Thompson, "Single-antenna selection for MISO cognitive radio," in Proc. IET Seminar, Cognitive Radio and Software Defined Radio: Technologies and Techniques, September 2008, pp. 1-5.

[11] J. Zhou, J. Thompson, and I. Krikidis, "Multiple antennas selection for linear precoding MISO cognitive radio," in Proc. IEEE WCNC 2009, April 2009, pp. 1-6. 